\title{
Robust Estimation for Discrete Markov System with Time-Varying Delay and Missing Measurements
}

\author{
Jia You, ${ }^{1}$ Shen Yin, ${ }^{2}$ and Hamid Reza Karimi ${ }^{3}$ \\ ${ }^{1}$ Research Institute of Intelligent Control and Systems, Harbin Institute of Technology, Heilongjiang 150001, China \\ ${ }^{2}$ College of Information Sciences and Engineering, Bohai University, Liaoning 121000, China \\ ${ }^{3}$ Department of Engineering, Faculty of Engineering and Science, the University of Agder, 4898 Grimstad, Norway
}

Correspondence should be addressed to Shen Yin; shen.yin2011@googlemail.com

Received 15 July 2013; Accepted 22 August 2013

Academic Editor: Xudong Zhao

Copyright (C) 2013 Jia You et al. This is an open access article distributed under the Creative Commons Attribution License, which permits unrestricted use, distribution, and reproduction in any medium, provided the original work is properly cited.

\begin{abstract}
This paper addresses the $\mathscr{H}_{\infty}$ filtering problem for time-delayed Markov jump systems (MJSs) with intermittent measurements. Within network environment, missing measurements are taken into account, since the communication channel is supposed to be imperfect. A Bernoulli process is utilized to describe the phenomenon of the missing measurements. The original system is transformed into an input-output form consisting of two interconnected subsystems. Based on scaled small gain (SSG) theorem and proposed Lyapunov-Krasovskii functional (LKF), the scaled small gains of the subsystems are analyzed, respectively. New conditions for the existence of the $\mathscr{H}_{\infty}$ filters are established, and the corresponding $\mathscr{H}_{\infty}$ filter design scheme is proposed. Finally, a simulation example is provided to demonstrate the effectiveness of the proposed approach.
\end{abstract}

\section{Introduction}

In recent years, networked control systems (NCSs) have found a great deal of applications in a range of engineering areas [1-6]. Compared to traditional control systems, the NCSs hold many magnificent advantages, such as reduced power and weight requirements, low cost, improved flexibility and suppleness, high reliability, and facilitated system diagnosis and maintenance. Consequently, NCSs have drawn extensive investigation, and a great deal of significant results have been reported [7-12].

On the other hand, some unexpected phenomena appear in the networked environment, since the digital communication channels have been introduced, on which all measured signals are transmitted with limited capacity. Intermittent measurements and time-delay are widely emerged in many kinds of NCSs, which could always lead to poor performance even instability of the systems. Some research issues in networked environment, such as filtering problems have become much more complicated since most traditional approaches are proposed on the assumption that the communication channel between the physical plant and filter is perfect. In this sense, a great attention has been attracted to the filtering problems with limited capacity and led to many important results. To mention them, [13] addresses the filtering problem with communication delay, [14] investigates the $H_{\infty}$ filtering problem for nonlinear stochastic systems, and robust filtering problems subject to stochastic missing measurements which described by different methods are introduced in [15-17].

Based on the direct Lyapunov method, a great deal of stability criteria have been reported $[9,18]$. Meanwhile, an input-output approach has been introduced to analyze the stability of the systems by transforming the original system into a form of interconnection of two subsystems via employing a two-term approximation for the delayed variables [19]. Recently, Observing that the resulting performance largely depends on the error between the delayed variable and the approximation, an approach for estimating the delay by the upper and the lower delay bounds was introduced [20], of which the approximation error is much smaller than the error obtained by other one-term approaches [21, 22]. However, to the best of the authors' knowledge, corresponding results for time-delay MJSs with limited capacity channel have been relatively few, which motivates the research in this paper. 
In this paper, we investigate the $\mathscr{H}_{\infty}$ filtering problem for time-delayed MJSs with intermittent measurements. The communication channel between the plant and the filter is supposed to be imperfect. And the phenomenon of missing measurements is modeled by employing a stochastic variable satisfying Bernoulli random binary distribution. By using a new two-term approximation to the delay variables, the system is transformed into an interconnected systems comprised of two subsystems. Based on the scaled small gain (SSG) theorem and proposed Lyapunov-Krasovskii functional (LKF), sufficient conditions for the existence of the $\mathscr{H}_{\infty}$ filters and the corresponding $\mathscr{H}_{\infty}$ filter design method are proposed. Finally, a numerical example is provided to show effectiveness of the approach.

The remainder of this paper is organized as follows. In Section 2, the problem of $\mathscr{H}_{\infty}$ filtering is formulated for timedelayed MJSs with intermittent measurements; Section 3 presents the results for $\mathscr{H}_{\infty}$ performance analysis, and $\mathscr{H}_{\infty}$ filter design problem is solved in Section 4; a numerical example is provided to demonstrate the effectiveness of the design filtering technique in Section 5, and we conclude the paper with Section 6.

Notation. Throughout this paper, $\mathbb{R}^{n}$ represents the $n$ dimensional Euclidean space, $\mathbb{R}^{n \times m}$ is the set of all $n \times m$ real matrices, the superscripts " -1 " and " $T$," respectively, stand for the matrix inverse and matrix transpose. $\operatorname{Sym}\{A\}$ is the shorten notation for $A+A^{T}$, and the notation $P>0$ (resp., $P \geq$ 0 ), for $P \in \mathbb{R}^{n \times n}$ means that $P$ is real symmetric and positive definite (resp., semidefinite). The symmetric elements of the symmetric matrix is represented by an asterisk $(*)$, and the block-diagonal matrices are denoted by $\operatorname{diag}\{\cdots\} . \mathbf{G}_{1} \circ \mathbf{G}_{2}$ means the series connection of mapping $\mathbf{G}_{1}$ and $\mathbf{G}_{2} . E\{\cdot\}$ denotes the expectation operator with respect to probability measure, and for vector $x(k),\|x\|_{E_{2}}=E\left\{\sum_{n=0}^{\infty}\|x(n)\|^{2}\right\}^{1 / 2}$.

\section{Problem Formulation}

Consider the following Markov jump system with the timevarying delay:

$$
\begin{gathered}
x_{k+1}=A\left(r_{k}\right) x_{k}+A_{d}\left(r_{k}\right) x_{k-\tau_{k}}+B\left(r_{k}\right) \omega_{k}, \\
y_{k}=C\left(r_{k}\right) x_{k}+C_{d}\left(r_{k}\right) x_{k-\tau_{k}}+D\left(r_{k}\right) \omega_{k}, \\
z_{k}=L\left(r_{k}\right) x_{k}, \\
x_{k}=\psi_{k}, \quad k=-d_{2}, \ldots,-1,0
\end{gathered}
$$

where $x_{k} \in \mathbb{R}^{n}$ denotes system state; $y_{k} \in \mathbb{R}^{m}$ is the measured output; $\omega_{k} \in \mathbb{R}^{q}$ is the measurement noise which belongs $L_{2}[0, \infty) ; z_{k} \in \mathbb{R}^{p}$ is the signal to be estimated; and $\psi_{k}$ is a given real-valued initial condition. Let $\left\{r_{k}, k \in \mathbb{Z}\right\}$ be a Markov chain that takes values in a given finite set $\mathcal{S}=$ $\{1,2, \ldots, s\}$ with transition probability $p_{i j}=\operatorname{Pr}\left\{r_{k+1}=j \mid\right.$ $\left.r_{k}=i\right\}$, where $p_{i j} \geq 0$ for $i, j \in \mathcal{S}$ and $\sum_{j=1}^{s} p_{i j}=1$. And $\tau_{k}$ is a positive integer, denoting the time-varying delay satisfying

$$
d_{1} \leq \tau(k) \leq d_{2}
$$

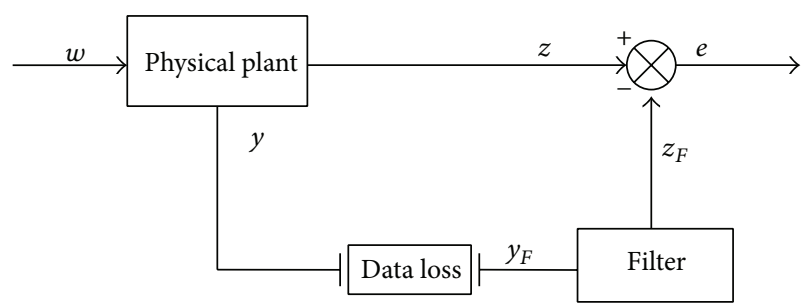

FIGURE 1: Filtering problem with intermittent measurements.

where $d_{1} \geq 0$ and $d_{2}>0$ are the minimum and the maximum delay, respectively. The delay interval is defined as $d_{t}=d_{2}-d_{1}$.

Then, we consider the following mode-independent filter with full order:

$$
\mathscr{F}:\left\{\begin{array}{l}
\widehat{x}_{k+1}=A_{F} \widehat{x}_{k}+B_{F} y_{F}(k), \\
\widehat{z}_{k}=L_{F} \widehat{x}_{k},
\end{array}\right.
$$

where $\widehat{x}_{k} \in \mathbb{R}^{n}$ is the filter state vector and $\widehat{z}_{k} \in \mathbb{R}^{p}$ is the output of the filter; $A_{F} \in \mathbb{R}^{n \times n}, B_{F} \in \mathbb{R}^{n \times m}$, and $C_{F} \in \mathbb{R}^{p \times n}$ are the filter gains to be determined.

In the network the data transmitted in the digital channel may be lost at a certain probability as shown in Figure 1. As a result, the output of the physical plant $y_{k}$ is no more equivalent to the input of the filter, which is denoted by $y_{F}(k)$. In this paper, this phenomenon is modeled via a Bernoulli process:

$$
y_{F}(k)=\alpha_{k} y_{k}
$$

where $\alpha_{k}$ is Bernoulli process, which models the intermittent transmission between the plant and the filter. When $\alpha(k)=1$, the data is transmitted to the filter successfully. While when $\alpha(k)=0$, the transmission fails and the data is lost. It can be described as:

$$
\operatorname{Prob}\left\{\alpha_{k}=1\right\}=E\left\{\alpha_{k}\right\}=\bar{\alpha}, \quad \operatorname{Prob}\left\{\alpha_{k}=0\right\}=1-\bar{\alpha} .
$$

Combining (3) and (5), we have

$$
\mathscr{F}:\left\{\begin{array}{l}
\widehat{x}_{k+1}=A_{F} \widehat{x}_{k}+B_{F} \alpha_{k} y_{k} \\
\widehat{z}_{k}=L_{F} \widehat{x}_{k} .
\end{array}\right.
$$

Defining $\widetilde{\alpha}=\alpha_{k}-\bar{\alpha}$ yields $E\{\widetilde{\alpha}\}=0$ and $E\left\{(\widetilde{\alpha})^{2}\right\}=\bar{\alpha}(1-\bar{\alpha})$. Then, denote

$$
\xi_{k}=\left[\begin{array}{ll}
x_{k}^{T} & \widehat{x}_{k}^{T}
\end{array}\right]^{T}, \quad e_{k}=z_{k}-\widehat{z}_{k}
$$

By connecting (1) with (3), the filtering error dynamic can be described by

$$
\mathcal{S}:\left\{\begin{array}{l}
\xi_{k+1}=\bar{A}(i) \xi_{k}+\bar{A}_{d}(i) K \xi_{k-\tau_{k}}+\bar{B}(i) \omega_{k}, \\
e_{k}=\bar{L}(i) \xi_{k},
\end{array}\right.
$$


where

$$
\begin{aligned}
& \bar{A}(i)=\bar{A}_{1}(i)+\widetilde{\alpha} \bar{A}_{2}(i), \quad \bar{A}_{d}(i)=\bar{A}_{d 1}(i)+\widetilde{\alpha} \bar{A}_{d 2}(i), \\
& \bar{B}(i)=\bar{B}_{1}(i)+\widetilde{\alpha} \bar{B}_{2}(i), \quad K=\left[\begin{array}{ll}
I & 0
\end{array}\right], \\
& \bar{A}_{1}(i)=\left[\begin{array}{cc}
A(i) & 0 \\
\bar{\alpha} B_{F} C(i) & A_{F}
\end{array}\right], \quad \bar{A}_{2}(i)=\left[\begin{array}{cc}
0 & 0 \\
B_{F} C(i) & 0
\end{array}\right], \\
& \bar{A}_{d 1}(i)=\left[\begin{array}{c}
A_{d}(i) \\
\bar{\alpha} B_{F} C_{d}(i)
\end{array}\right], \quad \bar{A}_{d 2}(i)=\left[\begin{array}{c}
0 \\
B_{F} C_{d}(i)
\end{array}\right], \\
& \bar{B}_{1}(i)=\left[\begin{array}{c}
B(i) \\
\alpha B_{F} D(i)
\end{array}\right], \quad \bar{B}_{2}(i)=\left[\begin{array}{c}
0 \\
B_{F} D(i)
\end{array}\right], \\
& \bar{L}(i)=\left[L(i)-L_{F}\right] .
\end{aligned}
$$

Moreover, we introduce the definition of stochastic stability which is vital and important in this paper.

Definition 1 (see [23]). For the system in (1) with $\omega_{k}=0$, the system is stochastically stable if for any $\psi_{k} \in \mathbb{R}^{n}$,

$$
E\left\{\sum_{k=0}^{\infty} x_{k}^{T} x_{k} \mid \psi(0)\right\}<\infty .
$$

Definition 2 (see [23]). A mapping G : $u_{k} \rightarrow y_{k}$ is inputoutput stable in mean-square if there exists $\gamma \geq 0$ such that

$$
\left\|y_{k}\right\|_{E_{2}}=\|\mathbf{G}(u)\|_{E_{2}} \leq \gamma\left\|u_{k}\right\|_{E_{2}} .
$$

Then, the $\mathscr{H}_{\infty}$ filtering design for MJSs with intermittent measurements to be addressed in this paper can be formulated as follows.

Consider system (1) with intermittent measurements modeled by (5). Given positive integers $d_{2}>d_{1}$ and a prescribed scalar $\gamma>0$, the filter gains $A_{F}, B_{F}$, and $L_{F}$ of filter (3) are designed such that for any time-delay $\tau_{k}$ satisfying (2), the filter error system (8) is stochastically stable, and $\|e\|_{E_{2}} \leq \gamma\|\omega\|_{E_{2}}$ holds under zero initial conditions.

\section{Main Results}

In this section, by developing SSG Theorem to stochastic systems and a new model transformation to system (8), we will focus on the filter performance analysis. Sufficient conditions are presented, under which system $\mathscr{E}$ is stochastically stable with a prescribed $\mathscr{H}_{\infty}$ performance index.

\subsection{Filtering Performance Analysis}

Lemma 3 (see [24]). One considers the following system consisting of two subsystems as shown in Figure 2:

$$
\mathscr{Y}_{1}: \zeta_{k}=\mathbf{G}\left(u_{k}-\delta_{k}\right), \quad \mathscr{Y}_{2}: \delta_{k}=\Delta \zeta_{k} .
$$

The closed loop system is input-output stable in meansquare for all the subsystems $\mathbf{G}$ and $\Delta$, if $\left\|T_{\zeta} \circ \mathbf{G} \circ T_{\delta}\right\|<1$ holds for some nonsingular matrices $T_{\zeta}$ and $T_{\delta}$ with $\left\|T_{\delta} \circ \Delta \circ T_{\zeta}\right\| \leq 1$.

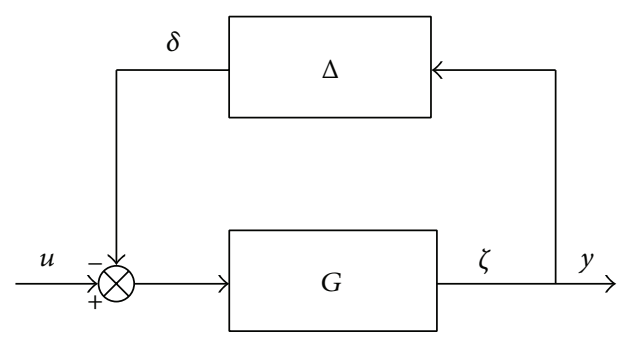

Figure 2: The interconnection subsystems.

Proof. According to (12) and the condition in Lemma 3, by defining $\Delta u_{k}=u_{k}-\delta_{k}$, and without loss of generality, it can be assumed that

$$
\begin{gathered}
\left\|\mathbf{G}\left(\Delta u_{k}\right)\right\|_{E_{2}} \leq k_{1}\left\|\Delta u_{k}\right\|_{E_{2}}, \quad\left\|\Delta\left(\zeta_{k}\right)\right\|_{E_{2}} \leq k_{2}\left\|\zeta_{k}\right\|_{E_{2}}, \\
0<k_{1}<1, \quad 0<k_{2} \leq 1 .
\end{gathered}
$$

Obviously, we have

$$
\begin{aligned}
\left\|T y_{k}\right\|_{E_{2}} & \leq k_{1}\left\|T \Delta u_{k}\right\|_{E_{2}}=k_{1}\left\|T\left(u_{k}-\Delta\left(y_{k}\right)\right)\right\|_{E_{2}} \\
& \leq k_{1}\left(\left\|T u_{k}\right\|_{E_{2}}+k_{2}\left\|T y_{k}\right\|_{E_{2}}\right), \\
& \left\|T y_{k}\right\|_{E_{2}} \leq \frac{k_{1}}{1-k_{1} k_{2}}\left\|T u_{k}\right\|_{E_{2}} .
\end{aligned}
$$

Since $k_{1} k_{2}<1$, system (12) is input-output stable in mean-square, which completes the proof.

Then, to separate the uncertainties $\tau_{k}$ from the filtering error system, the time-delay system $\mathcal{S}$ in (8) is transformed into the interconnection of two subsystems introduced in Lemma 3.

Notice that the time-varying delay can be written as

$$
x_{k-\tau_{k}}=\frac{1}{2} x_{k-d_{1}}+\frac{1}{2} x_{k-d_{2}}+\frac{d_{12}}{2} \delta_{k},
$$

where $(1 / 2)\left(x_{k-d_{1}}+x_{k-d_{2}}\right)$ is the approximation of the timevarying delay $x_{k-\tau_{k}}$, and $\left(d_{12} / 2\right) \delta_{k}$ is the approximation error. Then, we can describe system $\mathcal{S}$ as

$$
\mathcal{S}:\left\{\begin{array}{c}
\xi_{k+1}=\bar{A}(i) \xi_{k}+\frac{1}{2} \bar{A}_{d}(i) K \xi_{k-d_{1}}+\frac{1}{2} \bar{A}_{d}(i) K \xi_{k-d_{2}} \\
\quad+\frac{d_{12}}{2} \bar{A}_{d}(i) \delta_{k}+\bar{B}(i) \omega_{k}, \\
e_{k}=\bar{L}(i) \xi_{k} .
\end{array}\right.
$$

Defining $\zeta_{k}=x_{k+1}-x_{k}$ and

$$
\beta(i) \triangleq \begin{cases}1, & \text { when } i \leqslant k-\tau_{k}-1, \\ -1, & \text { when } i>k-\tau_{k}-1,\end{cases}
$$


we can obtain

$$
\begin{aligned}
\delta_{k} & =\frac{2}{d_{12}}\left[x_{k-\tau_{k}}-\frac{1}{2} x_{k-d_{1}}-\frac{1}{2} x_{k-d_{2}}\right] \\
& =\frac{1}{d_{12}}\left[\sum_{i=k-d_{2}}^{k-\tau_{k}-1} \zeta_{i}-\sum_{i=k-\tau_{k}}^{k-d_{1}-1} \zeta_{i}\right] \\
& =\frac{1}{d_{12}}\left[\sum_{i=k-d_{2}}^{k-d_{1}-1} \beta(i) \zeta_{i}\right] .
\end{aligned}
$$

From (18), we know that mapping $\Delta: \zeta_{k} \rightarrow \delta_{k}$ includes the uncertainty in $\tau_{k}$ leaving only two constant delays $d_{1}$ and $d_{2}$ in (16). Then the system in (16) can be described as

$$
\begin{gathered}
\mathcal{S}_{1}:\left[\begin{array}{c}
\xi_{k+1} \\
\zeta_{k} \\
e_{k}
\end{array}\right]=\underbrace{\left[\begin{array}{ccc}
\Phi_{1} & \frac{d_{12}}{2} \bar{A}_{d}(i) & \bar{B}(i) \\
\Phi_{2} & \frac{d_{12}}{2} A_{d}(i) & \bar{B}(i) \\
\Phi_{3} & 0 & 0
\end{array}\right]}_{\mathbf{G}}\left[\begin{array}{c}
\phi_{k} \\
\delta_{k} \\
\omega_{k}
\end{array}\right], \\
\mathcal{S}_{2}: \delta_{k}=\Delta \zeta_{k},
\end{gathered}
$$

where

$$
\begin{gathered}
\phi_{k}=\left[\begin{array}{lll}
\xi_{k}^{T} & \xi_{k-d_{1}}^{T} K^{T} & \xi_{k-d_{2}}^{T} K^{T}
\end{array}\right]^{T}, \\
\Phi_{1}=\left[\begin{array}{lll}
\bar{A}(i) & \frac{1}{2} \bar{A}_{d}(i) & \frac{1}{2} \bar{A}_{d}(i)
\end{array}\right], \\
\Phi_{2}=\left[\begin{array}{cll}
(A(i)-I) K & \frac{1}{2} A_{d}(i) & \frac{1}{2} A_{d}(i)
\end{array}\right], \\
\Phi_{3}=\left[\begin{array}{lll}
\bar{L} & 0 & 0
\end{array}\right] .
\end{gathered}
$$

Furthermore, the following lemma shows that the scaled small gain of the mapping $\Delta$ has an upper bound.

Lemma 4. $\gamma\left(\Delta_{T}\right)$ has an upper bound:

$$
\gamma\left(\Delta_{T}\right)=\sup \frac{\left\|T \delta_{k}\right\|_{E_{2}}}{\left\|T \zeta_{k}\right\|_{E_{2}}} \leq 1 .
$$

Proof. By using Jensen's inequality, it can be derived under zero initial condition according to the fact that $|\beta(i)|=1$,

$$
\begin{aligned}
\left\|T \delta_{k}\right\|_{E_{2}}^{2} & =E\left\{\sum_{j=0}^{\infty}\left[\delta_{j}^{T} Z \delta_{j}\right]\right\} \\
& \leq \frac{2}{d_{12}^{2}} E\left\{\sum_{i=0}^{\infty}\left[d_{12} \sum_{j=i-d_{2}}^{i-d_{1}-1} \beta^{2}(j) \delta_{j}^{T} \delta_{j}\right]\right\}
\end{aligned}
$$

$$
\begin{aligned}
& =\frac{1}{d_{12}} E\left\{\sum_{j=-d_{2} i=0}^{-d_{1}-1} \sum_{i+j}^{\infty} \delta_{i+j}^{T} \delta_{i}\right\} \\
& \leq \frac{1}{d_{12}} E\left\{\sum_{j=-d_{2} i=0}^{-d_{1}-1} \sum_{i}^{\infty} \delta_{i}^{T} \delta_{i}\right\} \\
& \leq E\left\{\sum_{j=0}^{\infty}\left[\zeta_{j}^{T} Z \zeta_{j}\right]\right\}=\left\|T \zeta_{k}\right\|_{E_{2}}^{2},
\end{aligned}
$$

where $Z=T^{T} T$, which implies $\gamma\left(\Delta_{T}\right) \leq 1$. The proof is completed.

Now, we are in the position to propose the sufficient conditions under which the filtering error system (8) is stochastically stable with a prescribed $\mathscr{H}_{\infty}$ performance attention level $\gamma$. First we assume the filter matrices $A_{F}, B_{F}$, and $L_{F}$ are fixed and the corresponding conditions are established in the following theorem.

Theorem 5. Consider system (1), and assume the filter matrices $A_{F}, B_{F}$, and $L_{F}$ are fixed. Given integers $d_{2}>d_{1} \geq 0$ and a constant $\gamma>0$, the filtering error system (16) is input-output stable in mean-square with an $\mathscr{H}_{\infty}$ performance attention level $\gamma$ for any time-varying delay satisfying (2) if there exist matrices $P>0, Q_{i}>0(i=1,2), R_{i}>0(i=1,2), Z>0$ satisfying

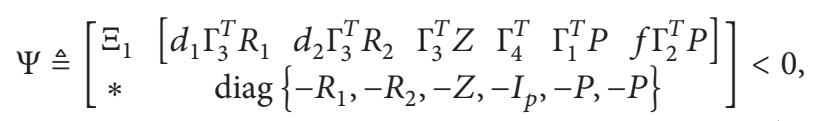

where

$$
\begin{aligned}
& \Xi_{1}=\left[\begin{array}{cc}
\Xi_{11} & {\left[\begin{array}{llll}
K^{T} R_{1} & K^{T} R_{2} & 0 & 0
\end{array}\right]} \\
* & \operatorname{diag}\left\{\Xi_{2}, \Xi_{3},-Z,-\gamma^{2} I_{q}\right\}
\end{array}\right], \\
& \Gamma_{1}=\left[\bar{A}_{1}(i) \frac{1}{2} \bar{A}_{d 1}(i) \frac{1}{2} \bar{A}_{d 1}(i) \frac{1}{2} d_{12} \bar{A}_{d 1}(i) \bar{B}_{1}(i)\right], \\
& \Gamma_{2}=\left[\bar{A}_{2}(i) \frac{1}{2} \bar{A}_{d 2}(i) \frac{1}{2} \bar{A}_{d 2}(i) \frac{1}{2} d_{12} \bar{A}_{d 2}(i) \bar{B}_{2}(i)\right], \\
& \Gamma_{3}=\left[(A(i)-I) K \frac{1}{2} A_{d}(i) \frac{1}{2} A_{d}(i) \frac{1}{2} d_{12} A_{d}(i) B(i)\right], \\
& \Gamma_{4}=\left[\begin{array}{ll}
\bar{L}(i) & 0_{p \times(3 n+q)}
\end{array}\right], \quad f=\sqrt{\bar{\eta}(1-\bar{\eta})}, \\
& \Xi_{11}=-P(i)+K^{T}\left(Q_{1}+Q_{2}-R_{1}-R_{2}\right) K, \\
& \Xi_{2}=-Q_{1}-R_{1}, \quad \Xi_{3}=-Q_{2}-R_{2} .
\end{aligned}
$$

Proof. Choose the following Lyapunov-Krasovskii functional:

$$
V(k)=V_{1}(k)+V_{2}(k)+V_{3}(k),
$$


with

$$
\begin{gathered}
V_{1}(k)=\xi_{k}^{T} P(i) \xi_{k}, \\
V_{2}(k)=\sum_{i=k-d_{1}}^{k-1} \xi_{i}^{T} K^{T} Q_{1} K \xi_{i}+\sum_{i=k-d_{2}}^{k-1} \xi_{i}^{T} K^{T} Q_{2} K \xi_{i}, \\
V_{3}(k)=d_{1} \sum_{i=-d_{1}}^{-1} \sum_{j=k+i}^{k-1} \zeta_{j}^{T} R_{1} \zeta_{j}+d_{2} \sum_{i=-d_{2}}^{-1} \sum_{j=k+i}^{k-1} \zeta_{j}^{T} R_{2} \zeta_{j},
\end{gathered}
$$

where $P>0$ and $Q_{i}>0$ are the matrices to be determined.

Defining $\Delta V(k)=E\{V(k+1)\}-V(k)$, we have

$$
\begin{aligned}
\Delta V_{1}(k)= & E\left\{\xi_{k+1}^{T} P\left(i^{+}\right) \xi_{k+1}\right\}-\xi_{k} P(i) \xi_{k} \\
= & \varphi_{k}^{T}\left(\Gamma_{1}^{T} P\left(i^{+}\right) \Gamma_{1}+f^{2} \Gamma_{2}^{T} P\left(i^{+}\right) \Gamma_{2}\right) \varphi_{k}-\xi_{k} P(i) \xi_{k}, \\
\Delta V_{2}(k)= & \xi_{k}^{T} K^{T} Q_{1} K \xi_{k}-\xi_{k-d_{1}}^{T} K^{T} Q_{1} K \xi_{k-d_{1}} \\
& +\xi_{k}^{T} K^{T} Q_{2} K \xi_{k}-\xi_{k-d_{2}}^{T} K^{T} Q_{2} K \xi_{k-d_{2}}, \\
\Delta V_{3}(k)= & d_{1}^{2} \zeta_{k}^{T} R_{1} \zeta_{k}+d_{2}^{2} \zeta_{k}^{T} R_{2} \zeta_{k} \\
& -d_{1} \sum_{j=k-d_{1}}^{k-1} \zeta_{j}^{T} R_{1} \zeta_{j}-d_{2} \sum_{j=k-d_{2}}^{k-1} \zeta_{j}^{T} R_{2} \zeta_{j},
\end{aligned}
$$

where $\varphi_{k}=\left[\begin{array}{llllll}\xi_{k}^{T} & \xi_{k-d_{1}}^{T} K^{T} & \xi_{k-d_{2}}^{T} K^{T} & \delta_{k}^{T} & \omega_{k}^{T}\end{array}\right]^{T}$.

Moreover, define

$$
J_{s}=E\left\{\sum_{k=0}^{\infty}\left(y_{k}^{T} Z y_{k}-\delta_{k}^{T} Z \delta_{k}+e_{k}^{T} e_{k}-\gamma^{2} \omega_{k}^{T} \omega_{k}\right)\right\} .
$$

Together with (25), and according to $V(0)=0$ and $V(\infty) \geq 0$, we have

$$
\begin{gathered}
J_{s} \leq J_{s}+V(\infty)-V(0) \\
=E\left\{\sum _ { k = 0 } ^ { \infty } \left(\Delta V(k)+\zeta_{k}^{T} Z \zeta_{k}-\delta_{k}^{T} Z \delta_{k}\right.\right. \\
\left.\left.+e_{k}^{T} e_{k}-\gamma^{2} \omega_{k}^{T} \omega_{k}\right)\right\} \\
=E\left\{\sum _ { k = 0 } ^ { \infty } \varphi _ { k } ^ { T } \left(\Xi_{1}+\Gamma_{3}^{T}\left(d_{1}^{2} R_{1}+d_{2}^{2} R_{2}+Z\right) \Gamma_{3}+\Gamma_{4}^{T} \Gamma_{4}\right.\right. \\
\left.\left.+\Gamma_{1}^{T} P\left(i^{+}\right) \Gamma_{1}+f \Gamma_{2}^{T} P\left(i^{+}\right) \Gamma_{2}\right) \varphi_{k}\right\},
\end{gathered}
$$
0 , if

It is obvious that $\Delta V(k)+\zeta_{k}^{T} Z \zeta_{k}-\delta_{k}^{T} Z \delta_{k}+e_{k}^{T} e_{k}-\gamma^{2} \omega_{k}^{T} \omega_{k}<$

$$
\begin{aligned}
\Xi_{1} & +\Gamma_{3}^{T}\left(d_{1}^{2} R_{1}+d_{2}^{2} R_{2}+Z\right) \Gamma_{3}+\Gamma_{4}^{T} \Gamma_{4}+\Gamma_{1}^{T} P\left(i^{+}\right) \Gamma_{1} \\
& +f \Gamma_{2}^{T} P\left(i^{+}\right) \Gamma_{2}<0,
\end{aligned}
$$

which, by Schur complement, is equivalent to (23). Therefore, defining $Z=T^{T} T$, we have $J_{s}<0$, if (23) holds, which means

$$
\left\|T \zeta_{k}\right\|_{E_{2}}^{2}+\left\|e_{k}\right\|_{E_{2}}^{2} \leq\left\|T \delta_{k}\right\|_{E_{2}}^{2}+\gamma^{2}\left\|\omega_{k}\right\|_{E_{2}}^{2},
$$

if Theorem 5 holds, which means the SSG of subsystem $\mathcal{S}_{1}$ satisfying $\gamma\left(\mathbf{G}_{T}\right)<1$. By Lemmas 3 and 4 , the filtering error system (16) is input-output stable in mean-square. Furthermore, we can obtain $\left\|e_{k}\right\|_{E_{2}}^{2} \leq \gamma^{2}\left\|\omega_{k}\right\|_{E_{2}}^{2}$ from (31) together with (22). The proof is completed.

Theorem 5 guarantees the input-output stability in meansquare of system in (19). Through the following research, it can be proved that the system in (19) is stochastically stable by Lyapunov-Krasovskii functional method.

Theorem 6. For the forward system $\mathcal{S}_{1}$ in (19) with $\omega_{k}=0$, if there exists an $L K F V(k)$ and $Z>0$ satisfying $\Delta V(k)+\zeta_{k}^{T} Z \zeta_{k}-$ $\delta_{k}^{T} Z \delta_{k}<0$, then the system in (19) is stochastically stable by the following $L K F$ :

$$
V_{c}(k)=V(k)+\frac{1}{d_{12}} \sum_{i=-d_{2}}^{-d_{1}-1} \sum_{j=k+i}^{k-1} \zeta_{k}^{T} Z \zeta_{k} .
$$

Proof. Defining $\Delta V_{c}(k)=V_{c}(k+1)-V_{c}(k)$; we have

$$
\begin{aligned}
\Delta V_{c}(k)= & \Delta V(k)+\frac{1}{d_{12}} \sum_{i=-d_{2}}^{-d_{1}-1}\left[\zeta_{k}^{T} Z \zeta_{k}-\zeta_{k+i}^{T} Z \zeta_{k+i}\right] \\
= & \Delta V(k)+\zeta_{k}^{T} Z \zeta_{k}-\frac{1}{d_{12}} \sum_{i=k-d_{2}}^{k-d_{1}-1}\left[\beta(i) \zeta_{i}^{T} Z \beta(i) \zeta_{i}\right], \\
\leq & \Delta V(k)+\zeta_{k}^{T} Z \zeta_{k} \\
& -\frac{1}{d_{12}^{2}}\left[\sum_{i=k-d_{2}}^{k-d_{1}-1} \beta(i) \zeta_{i}\right]^{T} Z\left[\sum_{i=k-d_{2}}^{k-d_{1}-1} \beta(i) \zeta_{i}\right] \\
= & \Delta V(k)+\zeta_{k}^{T} Z \zeta_{k}-\delta_{k}^{T} Z \delta_{k}<0,
\end{aligned}
$$

which is implied by (23). Let $\lambda>0$, we have

$$
\Delta V_{c}(k)=E\left\{V_{c}(k+1)\right\}-V_{c}(k) \leq-\theta x_{k}^{T} x_{k}<0 .
$$

By summing up the inequality on both sides from $k=$ $0, \ldots, R$, it can be obtained that

$$
E\left\{V_{c}(R)\right\}-V_{c}(0) \leq-\theta \sum_{k=0}^{R} x_{k}^{T} x_{k}<0 .
$$

When $R \rightarrow \infty$, we have

$$
\begin{aligned}
\theta\left\|x_{k}^{T} x_{k}\right\|_{E_{2}}^{2} & \leq V_{c}(0)-E\left\{V_{c}(\infty)\right\} \\
& \leq V_{c}(0)<\infty,
\end{aligned}
$$

which implies that the systems in (19) are stochastically stable. The proof is completed. 


\section{4. $\mathscr{H}_{\infty}$ Filter Design}

Theorem 7. Consider the Markov jump system (1). For given integers $d_{2}>d_{1} \geq 0$ and a constant $\gamma>0$, if there exist matrices $R_{i}, S, Q_{1}>0, Q_{2}>0, R_{1}>0, R_{2}>0, Z>0$, $\bar{A}_{F}, \bar{B}_{F}$, and $\bar{L}_{F}$ such that

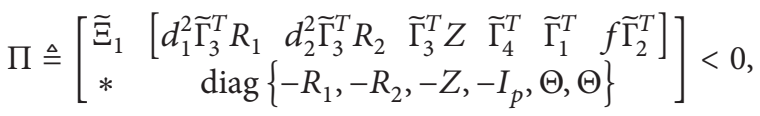

$$
\begin{aligned}
& S-R_{i}<0,
\end{aligned}
$$

where

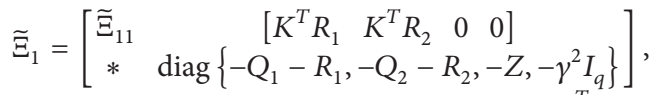

$$
\begin{aligned}
& \widetilde{\Gamma}_{1}=\left[\Gamma_{A}^{T} R+\bar{\eta} \Gamma_{F 1}^{T}+\Gamma_{F 2}^{T} \Gamma_{A}^{T} S+\bar{\eta} \Gamma_{F 1}^{T}+\Gamma_{F 2}^{T}\right]^{T},
\end{aligned}
$$

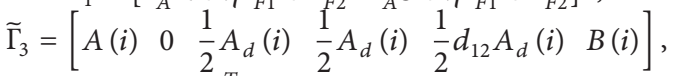

$$
\begin{aligned}
& \widetilde{\Gamma}_{2}=\left[\begin{array}{ll}
\Gamma_{F 1}^{T} & \Gamma_{F 1}^{T}
\end{array}\right]^{T}, \quad \widetilde{\Gamma}_{4}=\left[\begin{array}{lll}
L(i) & -L_{F} & 0_{p \times(3 n+q)}
\end{array}\right],
\end{aligned}
$$

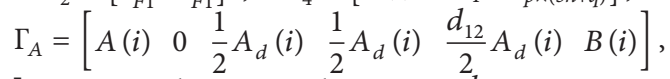

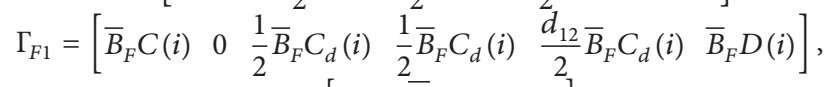

$$
\begin{aligned}
& \Gamma_{F 2}=\left[\begin{array}{lll}
0_{n \times n} & \bar{A}_{F} & 0_{n \times(3 n+q)}
\end{array}\right], \\
& \widetilde{\Xi}_{11}=-\widetilde{P}(i)+K^{T}\left(Q_{1}+Q_{2}-R_{1}-R_{2}\right) K \text {, } \\
& \Theta=\left[\begin{array}{cc}
\widetilde{R} & -S \\
-S & -S
\end{array}\right], \quad \widetilde{R}=-\sum_{i=1}^{s} p_{i j} R_{i},
\end{aligned}
$$

then, there exists an $\mathscr{H}_{\infty}$ filter of the type (3) such that the filtering error system in (8) is stochastically stable with a prescribed $\mathscr{H}_{\infty}$ performance level $\gamma$. Furthermore, the filter gains are given by

$$
\left[\begin{array}{cc}
A_{F} & B_{F} \\
L_{F} & 0
\end{array}\right]=\left[\begin{array}{cc}
S^{-1} & 0 \\
0 & I
\end{array}\right]\left[\begin{array}{cc}
\bar{A}_{F} & \bar{B}_{F} \\
\bar{L}_{F} & 0
\end{array}\right] .
$$

Proof. Suppose there exist matrices $P(i)>0, Q_{i}>0, R_{i}>0$, $(i=1,2), Z>0, A_{F}, B_{F}$, and $L_{F}$ satisfying (23). Partition $P$ as

$$
P(i) \triangleq\left[\begin{array}{cc}
P_{1 i} & P_{2} \\
P_{2}^{T} & P_{3}
\end{array}\right]
$$

and define matrices $J$ and $J_{1}$ as follows:

$$
\begin{gathered}
J \triangleq\left[\begin{array}{cc}
I & 0 \\
0 & P_{3}^{-1} P_{2}^{T}
\end{array}\right], \\
J_{1} \triangleq \operatorname{diag}\{J, I, I, I, I, I, I, J, J\} .
\end{gathered}
$$

According to the construction of $J$ and $J_{1}$ and the fact that $P_{2}$ and $P_{3}$ are nonsingular even disturbed by a small perturbation, it is obvious that matrix $J$ is invertible. Let

$$
R_{i} \triangleq P_{1 i}, \quad S \triangleq P_{2} P_{3}^{-1} P_{2}^{T} .
$$

Then, multiply (23) by $J_{1}^{T}$ from the left side and its transpose from the right side, respectively, and define

$$
\left[\begin{array}{cc}
\bar{A}_{F} & \bar{B}_{F} \\
\bar{L}_{F} & 0
\end{array}\right] \triangleq\left[\begin{array}{cc}
P_{2} & 0 \\
0 & I
\end{array}\right]\left[\begin{array}{cc}
A_{F} & B_{F} \\
L_{F} & 0
\end{array}\right]\left[\begin{array}{cc}
P_{3}^{-1} P_{2}^{T} & 0 \\
0 & I
\end{array}\right],
$$

we obtain (37).
On the other hand, suppose that matrices $R_{i}>0, S>0$, $Q_{1}>0, Q_{2}>0, R_{1}>0, R_{2}>0, Z>0, \bar{A}_{F}, \bar{B}_{F}$, and $\bar{L}_{F}$ satisfy (37). Introduce $P_{1 i} \triangleq R_{i}$ and nonsingular square matrices $P_{2}$ and $P_{3}$ satisfying $S=P_{2} P_{3}^{-1} P_{2}^{T}$. By Schur complement, $P(i)>$ 0 is equivalent to $R_{i}>0$ and $R_{i}-S=P_{1 i}-P_{2} P_{3}^{-1} P_{2}^{T}>0$. Then, without loss of generality, setting $P_{2}^{-T} P_{3}=I$ yields (39). The proof is completed.

\section{Numerical Example}

In this section, we provide an example to demonstrate the effectiveness of the filter design method proposed in the preceding sections.

Consider system (1) with the following parameters:

$$
\begin{aligned}
& A_{1}=\left[\begin{array}{ccc}
-0.1 & 0 & 0.01 \\
-0.59 & -0.1 & 0.02 \\
0.1 & -0.06 & -0.1
\end{array}\right] \text {, } \\
& A_{d 1}=\left[\begin{array}{ccc}
0.01 & 0.02 & 0 \\
0.01 & 0 & 0.01 \\
0 & 0 & -0.01
\end{array}\right] \text {, } \\
& B_{1}=\left[\begin{array}{lll}
-0.202 & 0.383 & 0.139
\end{array}\right]^{T} \text {, } \\
& C_{1}=\left[\begin{array}{ccc}
-0.02 & -0.01 & -0.02 \\
0.05 & 0.02 & 0.021
\end{array}\right] \text {, } \\
& C_{d 1}=\left[\begin{array}{ccc}
-0.01 & 0 & -0.02 \\
0.01 & 0.02 & 0
\end{array}\right] \text {, } \\
& L_{1}=\left[\begin{array}{lll}
0.2 & 0.5 & 0.1
\end{array}\right], \quad D_{1}=\left[\begin{array}{ll}
0.1 & 0.47
\end{array}\right]^{T} \text {, } \\
& A_{2}=\left[\begin{array}{ccc}
-0.1 & 0.1 & 0.04 \\
-0.51 & -0.21 & 0.01 \\
0.1 & -0.04 & -0.1
\end{array}\right] \text {, } \\
& A_{d 2}=\left[\begin{array}{ccc}
0.01 & 0.02 & 0 \\
0.01 & 0 & 0.01 \\
0 & 0 & 0.01
\end{array}\right] \text {, } \\
& B_{2}=\left[\begin{array}{lll}
-0.134 & 0.248 & 0.117
\end{array}\right]^{T} \text {, } \\
& C_{2}=\left[\begin{array}{ccc}
0.01 & -0.04 & 0.015 \\
0.031 & 0.042 & 0.071
\end{array}\right] \text {, } \\
& C_{d 2}=\left[\begin{array}{lll}
0.01 & 0 & -0.01 \\
0.02 & 0 & -0.01
\end{array}\right] \text {, } \\
& L_{2}=\left[\begin{array}{lll}
0.1 & 0.3 & 0.2
\end{array}\right], \quad D_{2}=\left[\begin{array}{ll}
0.23 & 0.15
\end{array}\right]^{T} \text {, }
\end{aligned}
$$

and the mode switching is governed by a Markov chain with transition probability:

$$
\begin{array}{ll}
p_{11}=0.8, & p_{12}=0.2 ; \\
p_{21}=0.7, & p_{22}=0.3 .
\end{array}
$$

Suppose $d_{1}=0$ and $d_{2}=1$ and the initial condition $x(0)=\left[\begin{array}{lll}0 & 0 & 0\end{array}\right]^{T}, x_{F}(0)=\left[\begin{array}{ll}0 & 0\end{array}\right]^{T}$. Then, by Theorem $7, \mathrm{a}$ filter in the form of (3) is designed such that the system (8) is stochastically stable with a guaranteed $\mathscr{H}_{\infty}$ performance level $\gamma$. 
Suppose the packet loss parameter is $\bar{\eta}=0.8$ as shown in Figure 3. By (37), it can be obtained that the minimum $\mathscr{H}_{\infty}$ performance $\gamma_{\min }=0.2801$, and

$$
\begin{aligned}
& S=\left[\begin{array}{ccc}
0.6417 & 0.2409 & 0.4963 \\
0.2409 & 0.4799 & -0.2105 \\
0.4963 & -0.2105 & 0.9024
\end{array}\right], \\
& R_{1}=\left[\begin{array}{ccc}
3.5491 & 1.1436 & 1.4231 \\
1.1436 & 1.1211 & -0.3808 \\
1.4231 & -0.3808 & 2.9697
\end{array}\right] \text {, } \\
& R_{2}=\left[\begin{array}{lll}
4.4848 & 1.2366 & 4.0199 \\
1.2366 & 1.1842 & 0.0895 \\
4.0199 & 0.0895 & 6.1286
\end{array}\right], \\
& Q_{11}=\left[\begin{array}{lll}
0.2861 & 0.0567 & 0.2708 \\
0.0567 & 0.0132 & 0.0426 \\
0.2708 & 0.0426 & 0.3200
\end{array}\right], \\
& Q_{12}=\left[\begin{array}{lll}
0.6548 & 0.1010 & 0.8336 \\
0.1010 & 0.0191 & 0.1131 \\
0.8336 & 0.1131 & 1.1341
\end{array}\right], \\
& Q_{21}=\left[\begin{array}{lll}
0.3141 & 0.0677 & 0.2825 \\
0.0677 & 0.0164 & 0.0513 \\
0.2825 & 0.0513 & 0.3075
\end{array}\right] \text {, } \\
& Q_{22}=\left[\begin{array}{lll}
0.6109 & 0.0934 & 0.7752 \\
0.0934 & 0.0174 & 0.1047 \\
0.7752 & 0.1047 & 1.0582
\end{array}\right] \text {, } \\
& R_{11}=\left[\begin{array}{lll}
0.3630 & 0.0516 & 0.2146 \\
0.0516 & 0.0116 & 0.0078 \\
0.2146 & 0.0078 & 0.2992
\end{array}\right] \text {, } \\
& R_{12}=\left[\begin{array}{lll}
0.6051 & 0.0888 & 0.8173 \\
0.0888 & 0.0196 & 0.1033 \\
0.8173 & 0.1033 & 1.1513
\end{array}\right] \text {, } \\
& R_{21}=\left[\begin{array}{lll}
0.1321 & 0.0183 & 0.0725 \\
0.0183 & 0.0035 & 0.0049 \\
0.0725 & 0.0049 & 0.0786
\end{array}\right], \\
& R_{22}=\left[\begin{array}{lll}
0.1288 & 0.0211 & 0.1679 \\
0.0211 & 0.0058 & 0.0214 \\
0.1679 & 0.0214 & 0.2356
\end{array}\right] \text {, } \\
& Z=\left[\begin{array}{lll}
0.1642 & 0.0233 & 0.2229 \\
0.0233 & 0.0085 & 0.0193 \\
0.2229 & 0.0193 & 0.3438
\end{array}\right],
\end{aligned}
$$

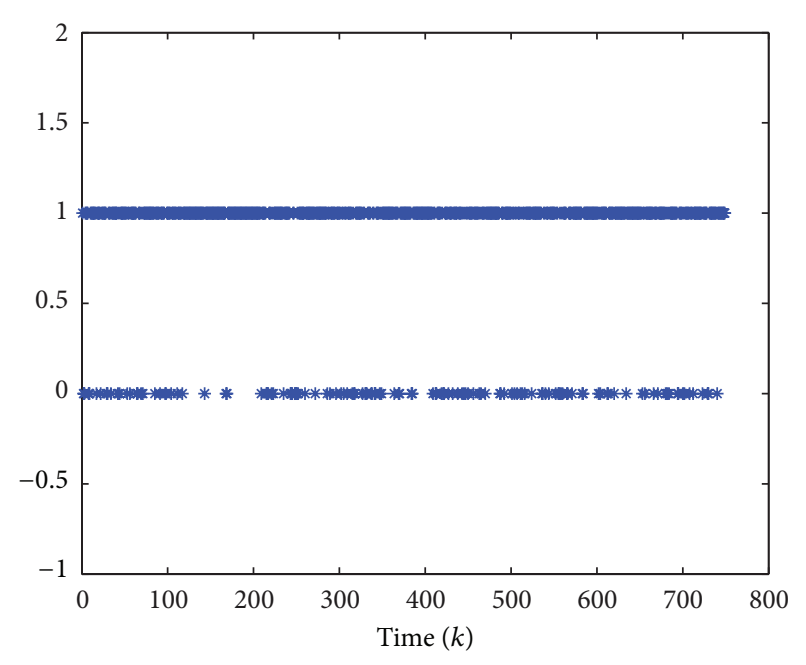

FIGURE 3: Packet loss parameter $\eta(k)$.

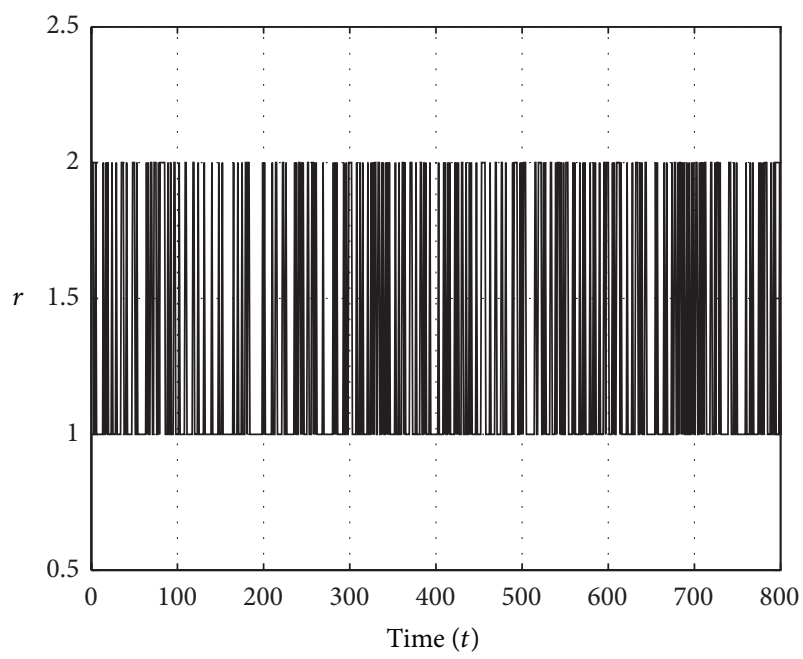

FIGURE 4: Switching states of Markov chain.

and the filter matrices are as follows:

$$
\begin{aligned}
& \bar{A}_{F}=\left[\begin{array}{ccc}
-0.2262 & -0.0798 & -0.0365 \\
-0.0678 & 0.0340 & -0.0505 \\
-0.1747 & -0.1394 & 0.0068
\end{array}\right], \\
& \bar{B}_{F}=\left[\begin{array}{cc}
0.0390 & -0.1099 \\
0.2651 & -0.0906 \\
-0.1568 & -0.0028
\end{array}\right], \\
& \bar{L}_{F}=\left[\begin{array}{lll}
-0.1391 & -0.4850 & 0.3233
\end{array}\right] .
\end{aligned}
$$

In addition, by (39), we have

$$
\begin{aligned}
A_{F} & =\left[\begin{array}{ccc}
-0.5488 & -0.0592 & 0.0027 \\
0.2024 & 0.0525 & -0.1157 \\
0.1555 & -0.1096 & -0.0210
\end{array}\right], \\
B_{F} & =\left[\begin{array}{cc}
-0.8717 & -0.4468 \\
1.2524 & 0.1581 \\
0.5979 & 0.2796
\end{array}\right], \\
\bar{L}_{F} & =\left[\begin{array}{lll}
-0.1391 & -0.4850 & 0.3233
\end{array}\right] .
\end{aligned}
$$




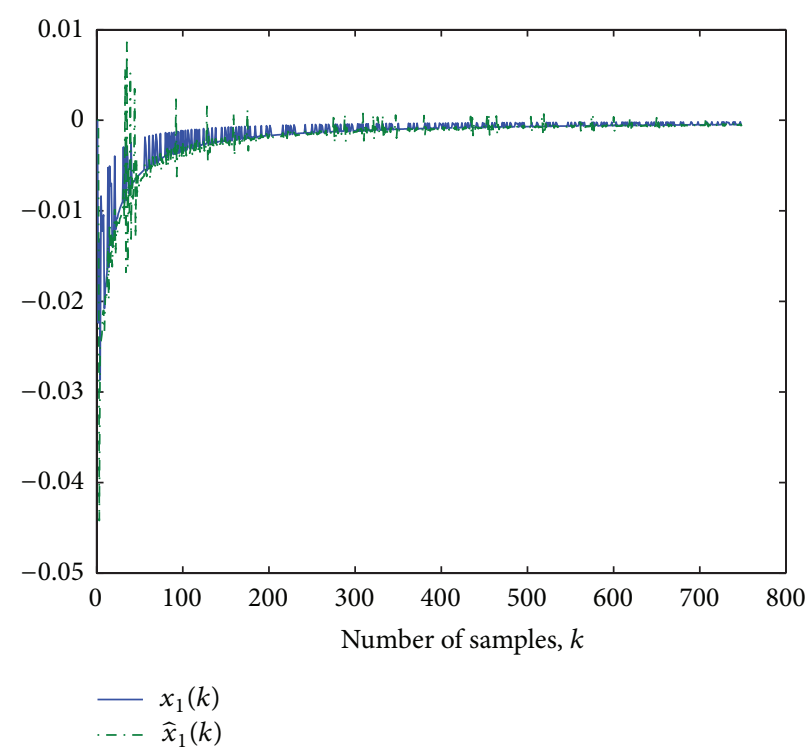

(a) $x_{1}$ and estimation $\widehat{x}_{1}$

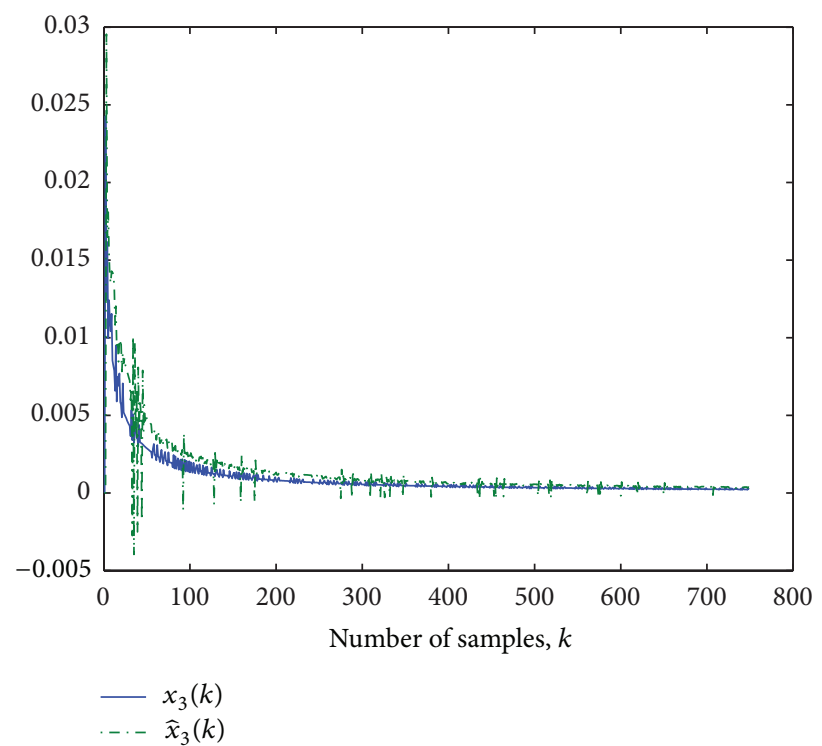

(c) $x_{3}$ and estimation $\widehat{x}_{3}$

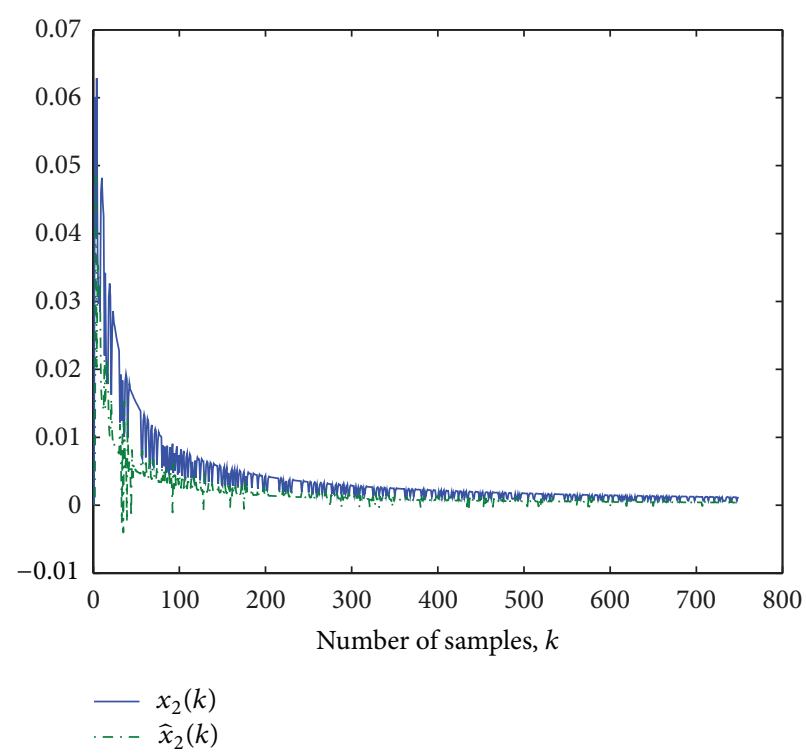

(b) $x_{2}$ and estimation $\hat{x}_{2}$

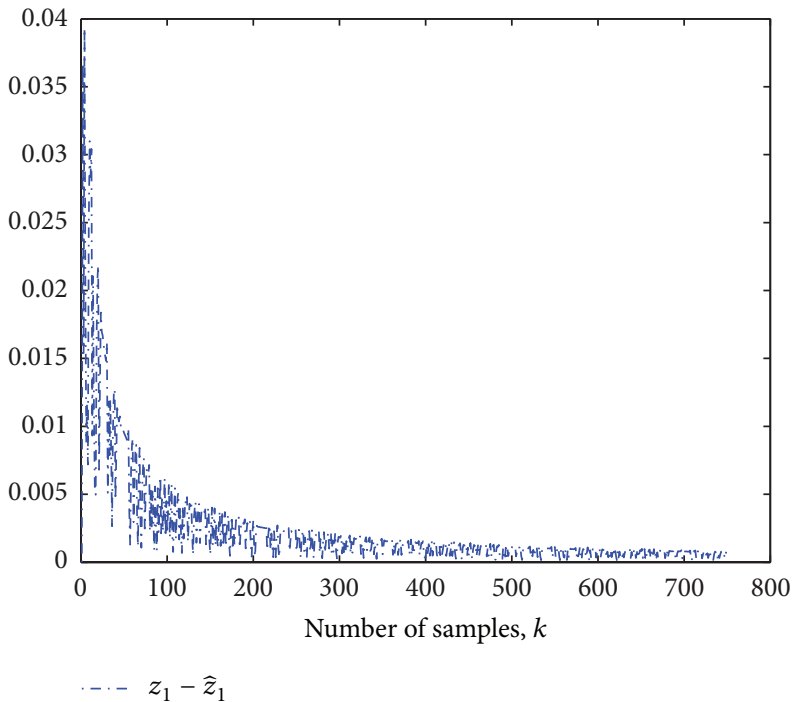

(d) signal $z_{1}-\widehat{z}_{1}$

FIGURE 5: Original signal and estimation signal.

To illustrate the performance of the filter, it is assumed that the external disturbance $\omega_{k}=2 /(k+10)$. By calculation, the switching of Markov chain is shown in Figure 4. Figure 5 shows the system state $x_{k}$ and its estimation $\widehat{x}_{k}$ and signal $z_{k}$ and its estimation $\widehat{z}_{k}$. Moreover, it can be obtained that the $\|e(k)\|_{E_{2}}=0.0166$ and $\|\omega(k)\|_{E_{2}}=0.3754$ as shown in Figure 5, that yields $\gamma=0.0626$ which is below the minimum $\gamma_{\min }=1.7051$, demonstrating the effectiveness of the proposed filter design.

\section{Conclusion}

This paper addresses the $\mathscr{H}_{\infty}$ filtering problem for time-delay Markov jump systems subject to intermittent measurements. The communication link failure from the plant to the filter is modeled by a stochastic variable satisfying the Bernoulli random binary distribution. The system is transformed into an input-output form consisted of two interconnected subsystems. Based on the SSG Theorem developed to stochastic systems and the proposed Lyapunov-Krasovskii function, sufficient conditions under which the $\mathscr{H}_{\infty}$ filter can be achieved with the prescribed $\mathscr{H}_{\infty}$ performance index is established. Finally, a numerical example is presented to demonstrate the effectiveness of the proposed filter design scheme.

\section{Conflict of Interests}

The authors declare that there is no conflict of interests regarding the publication of this paper. 


\section{Acknowledgment}

This work is supported by National Natural Science Foundation of China (Grant no. 61304102) and Natural Science Foundation of Liaoning Province of China (Grant no. 2013020002).

\section{References}

[1] H. Gao, W. Sun, and P. Shi, "Robust sampled-data $H_{\infty}$ control for vehicle active suspension systems," IEEE Transactions on Control Systems Technology, vol. 18, no. 1, pp. 238-245, 2010.

[2] P. Shi, E. Boukas, and R. K. Agarwal, "Kalman filtering for continuous-time uncertain systems with Markovian jumping parameters," IEEE Transactions on Automatic Control, vol. 44, no. 8, pp. 1592-1597, 1999.

[3] W. Sun, Y. Zhao, J. Li, L. Zhang, and H. Gao, "Active suspension control with frequency band constraints and actuator input delay," IEEE Transactions on Industrial Electronics, vol. 59, no. 1, pp. 530-537, 2012.

[4] L. Wu, X. Su, and P. Shi, "Sliding mode control with bounded $L_{2}$ gain performance of Markovian jump singular time-delay systems," Automatica, vol. 48, no. 8, pp. 1929-1933, 2012.

[5] S. Yin, S. Ding, A. Abandan Sari, and H. Hao, "Data-driven monitoring for stochastic systems and its application on batch process," International Journal of Systems Science, vol. 44, no. 7, pp. 1366-1376, 2013.

[6] S. Yin, H. Luo, and S. Ding, "Real-time implementation of faulttolerant control systems with performance optimization," IEEE Transactions on Industrial Electronics, 2013.

[7] M. Liu, D. W. C. Ho, and Y. Niu, "Stabilization of Markovian jump linear system over networks with random communication delay," Automatica, vol. 45, no. 2, pp. 416-421, 2009.

[8] M. Liu, D. W. C. Ho, and Y. Niu, "Robust filtering design for stochastic system with mode-dependent output quantization," IEEE Transactions on Signal Processing, vol. 58, no. 12, pp. 64106416, 2010.

[9] X. Meng, J. Lam, B. Du, and H. Gao, "A delay-partitioning approach to the stability analysis of discrete-time systems," Automatica, vol. 46, no. 3, pp. 610-614, 2010.

[10] L. Wu, P. Shi, H. Gao, and C. Wang, "A new approach to robust $H_{\infty}$ filtering for uncertain systems with both discrete and distributed delays," Circuits, Systems, and Signal Processing, vol. 26, no. 2, pp. 229-248, 2007.

[11] L. Wu, X. Yao, and W. Zheng, "Generalized $\mathrm{H}_{2}$ fault detection for two-dimensional Markovian jump systems," Automatica, vol. 48, no. 8, pp. 1741-1750, 2012.

[12] L. Wu and W. X. Zheng, "Passivity-based sliding mode control of uncertain singular time-delay systems," Automatica, vol. 45, no. 9, pp. 2120-2127, 2009.

[13] Z. Wang, F. Yang, D. W. C. Ho, and X. Liu, "Robust $H_{\infty}$ filtering for stochastic time-delay systems with missing measurements," IEEE Transactions on Signal Processing, vol. 54, no. 7, pp. 25792587, 2006.

[14] F. Yang, Z. Wang, D. W. C. Ho, and X. Liu, "Robust $H_{2}$ filtering for a class of systems with stochastic nonlinearities," IEEE Transactions on Circuits and Systems II, vol. 53, no. 3, pp. 235-239, 2006.

[15] Z. Wang, D. Ho, and X. Liu, "Variance-constrained filtering for uncertain stochastic systems with missing measurements," IEEE Transactions on Automatic Control, vol. 48, no. 7, pp. 1254-1258, 2003.
[16] Z. Wang, F. Yang, D. W. C. Ho, and X. Liu, "Robust finitehorizon filtering for stochastic systems with missing measurements," IEEE Signal Processing Letters, vol. 12, no. 6, pp. 437-440, 2005.

[17] D. Yue, Q. Han, and J. Lam, "Network-based robust $H_{\infty}$ control of systems with uncertainty," Automatica, vol. 41, no. 6, pp. 9991007, 2005.

[18] H. Gao and T. Chen, "New results on stability of discrete-time systems with time-varying state delay," IEEE Transactions on Automatic Control, vol. 52, no. 2, pp. 328-334, 2007.

[19] P. Bliman, "Extension of Popov absolute stability criterion to non-autonomous systems with delays," International Journal of Control, vol. 73, no. 15, pp. 1349-1361, 2000.

[20] C. Kao and B. Lincoln, "Simple stability criteria for systems with time-varying delays," Automatica, vol. 40, no. 8, pp. 1429-1434, 2004.

[21] K. Gu, Y. Zhang, and S. Xu, "Small gain problem in coupled differential-difference equations, time-varying delays, and direct Lyapunov method," International Journal of Robust and Nonlinear Control, vol. 21, no. 4, pp. 429-451, 2011.

[22] X. Li and H. Gao, "A new model transformation of discretetime systems with time timevarying delay and its application to stability analysis," IEEE Transactions on Automatic Control, no. 99, pp. 2172-2178, 2011.

[23] P. Seiler and R. Sengupta, "An $H_{\infty}$ approach to networked control," IEEE Transactions on Automatic Control, vol. 50, no. 3, pp. 356-364, 2005.

[24] K. Gu, V. Kharitonov, and J. Chen, Stability of Time-Delay Systems, Birkhäauser, Boston, Mass, USA, 2003. 


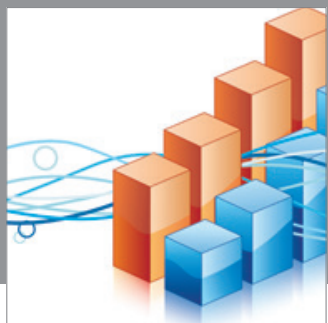

Advances in

Operations Research

mansans

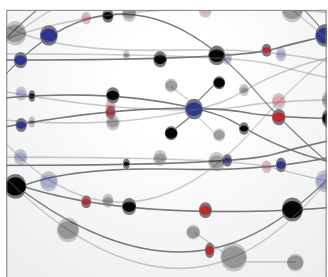

The Scientific World Journal
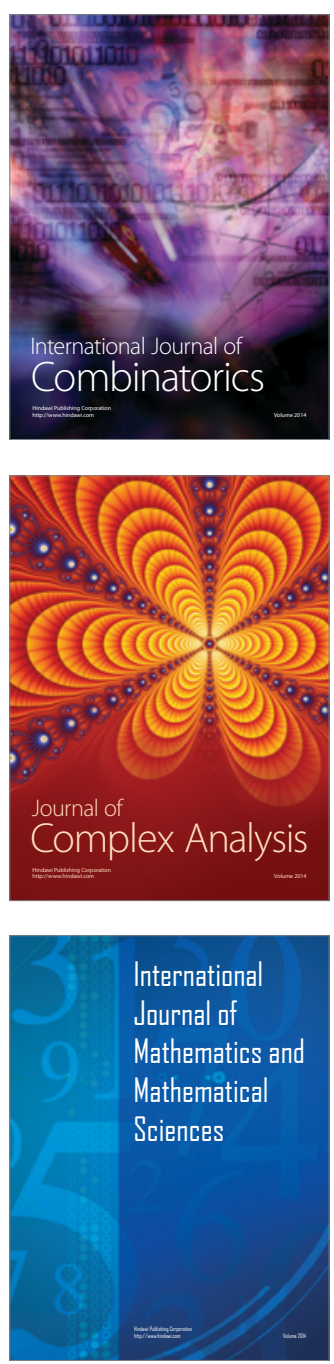
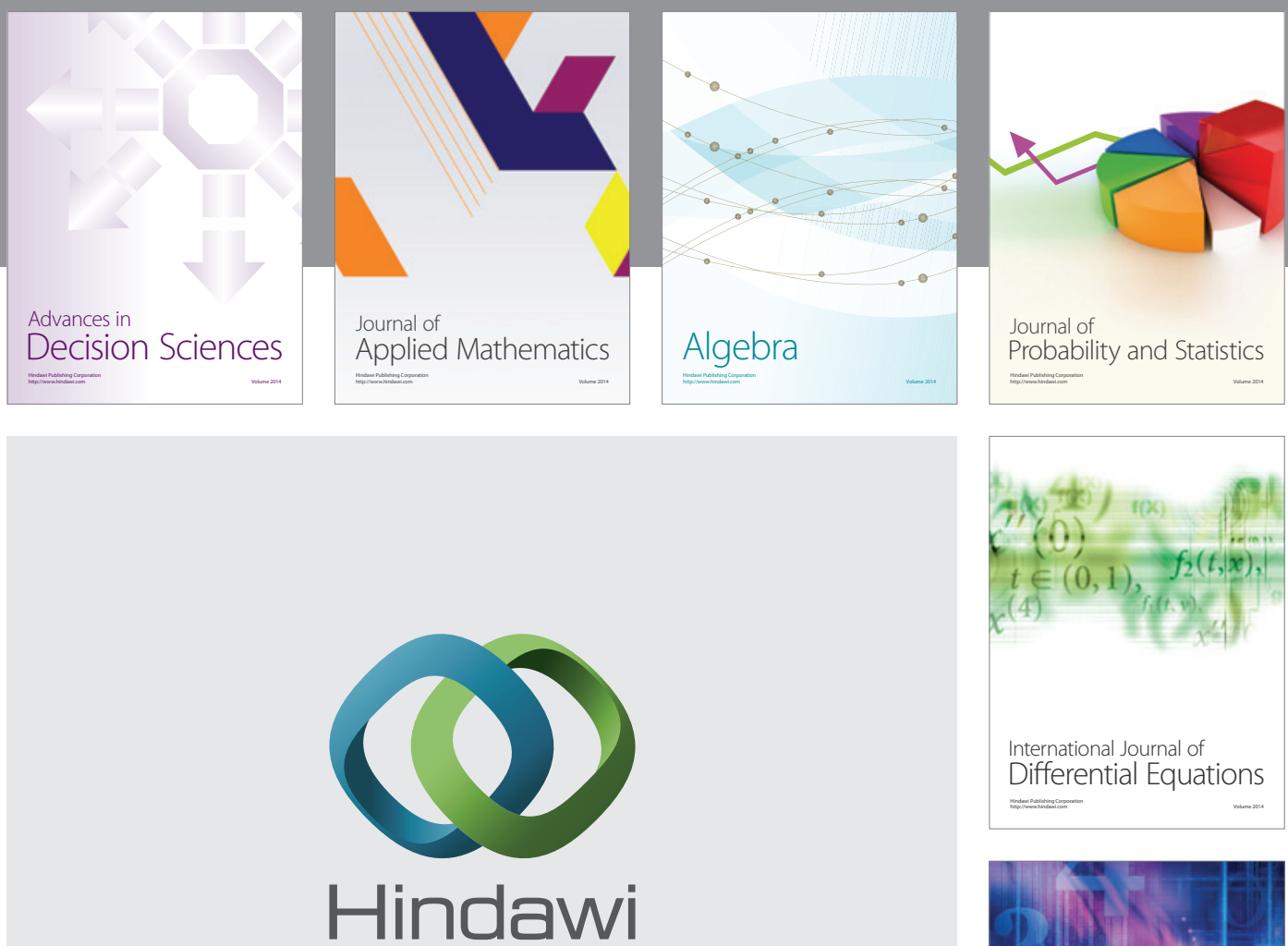

Submit your manuscripts at http://www.hindawi.com
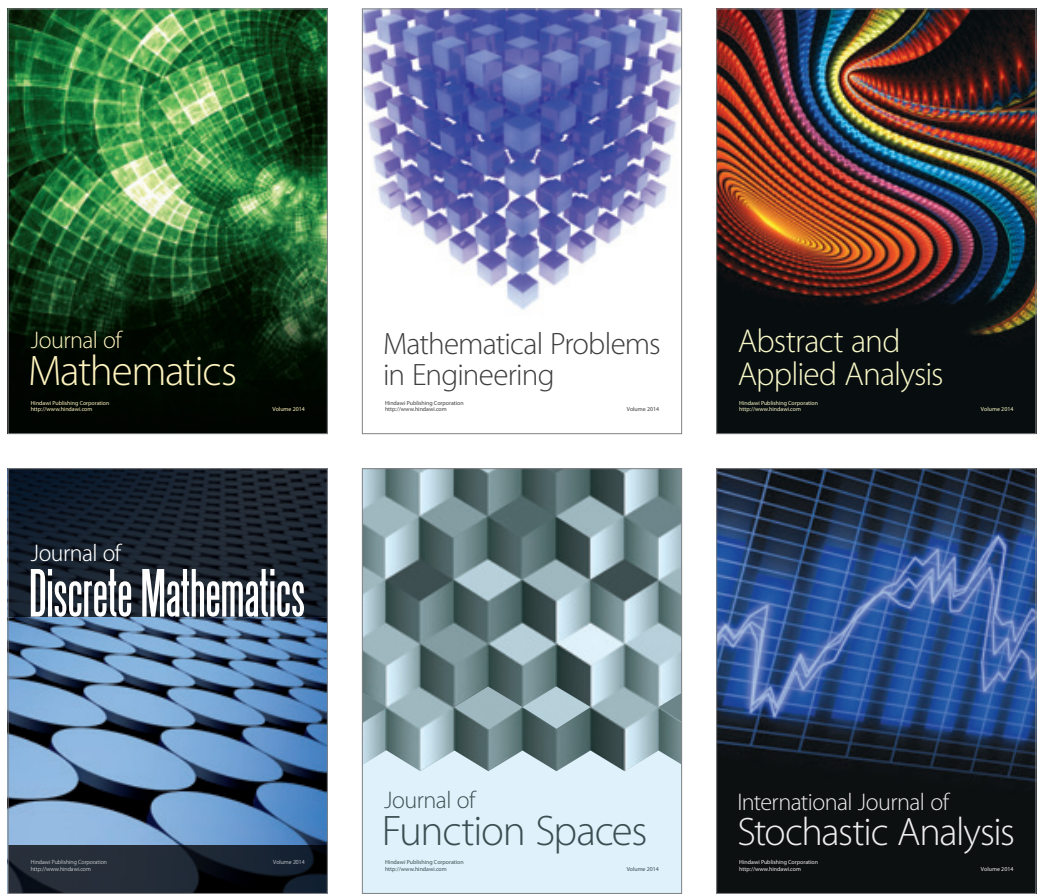

Journal of

Function Spaces

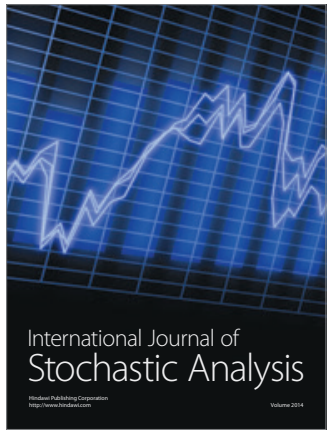

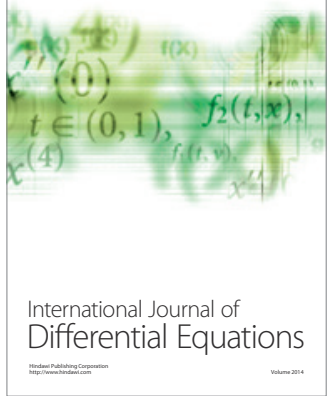
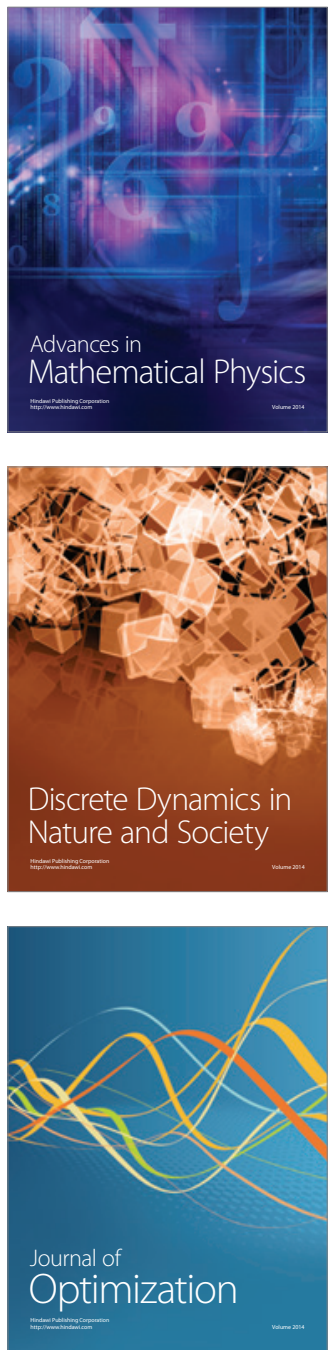